\title{
TECHNIQUES OF THE DIFFERENTIAL SUBORDINATION FOR DOMAINS BOUNDED BY CONIC SECTIONS
}

\author{
STANISŁAWA KANAS
}

Received 24 February 2003

\begin{abstract}
We solve the problem of finding the largest domain $D$ for which, under given $\psi$ and $q$, the differential subordination $\psi\left(p(z), z p^{\prime}(z)\right) \in D \Rightarrow p(z) \prec q(z)$, where $D$ and $q(U)$ are regions bounded by conic sections, is satisfied. The shape of the domain $D$ is described by the shape of $q(U)$. Also, we find the best dominant of the differential subordination $p(z)+\left(z p^{\prime}(z) /(\beta p(z)+\gamma)\right) \prec p_{k}(z)$, when the function $p_{k}(k \in[0, \infty))$ maps the unit disk onto a conical domain contained in a right half-plane. Various applications in the theory of univalent functions are also given.
\end{abstract}

2000 Mathematics Subject Classification: 30C45, 34A25, 33E05, 30C35.

1. Introduction. For $k \in[0, \infty)$ define the domain $\Omega_{k}$ as follows:

$$
\Omega_{k}=\left\{u+i v: u^{2}>k^{2}(u-1)^{2}+k^{2} v^{2}\right\} .
$$

For fixed $k$ the above domain represents the conic region bounded, successively, by the imaginary axis $(k=0)$, the right branch of a hyperbola $(0<k<1)$, a parabola $(k=1)$, and an ellipse $(k>1)$. Also we note that, for no choice of parameter $k(k>1), \Omega_{k}$ reduces to a disk. We present below the univalent functions mapping the unit disk $u$ onto $\Omega_{k}$, denoted by $p_{k}$, such that $p_{k}(0)=1$ and $p_{k}^{\prime}(0)>0$ :

$$
p_{k}(z)= \begin{cases}\frac{1+z}{1-z}, & \text { for } k=0, \\ 1+\frac{2}{1-k^{2}} \sinh ^{2}(A(k) \operatorname{arctanh} \sqrt{z}), & \text { for } k \in(0,1), \\ 1+\frac{2}{\pi^{2}} \log ^{2} \frac{1+\sqrt{z}}{1-\sqrt{z}}, & \text { for } k=1, \\ 1+\frac{2}{k^{2}-1} \sin ^{2}\left(\frac{\pi}{2 \mathscr{K}(t)} \mathscr{F}\left(\frac{\sqrt{z}}{\sqrt{t}}, t\right)\right), & \text { for } k>1,\end{cases}
$$

where $A(k)=(2 / \pi) \arccos k, \mathscr{F}(w, t)$ is the Legendre elliptic integral of the first kind,

$$
\mathscr{F}(w, t)=\int_{0}^{w} \frac{d x}{\sqrt{1-x^{2}} \sqrt{1-t^{2} x^{2}}}, \quad \mathscr{K}(t)=\mathscr{F}(1, t),
$$

and $t \in(0,1)$ is chosen such that $k=\cosh \left(\pi \mathcal{K}^{\prime}(t) /(2 \mathscr{K}(t))\right)$. 
The function $p_{0}$ is well known; an explicit form of $p_{1}$ is due to Rønning [16] and independently Ma and Minda [11]; the remaining cases belong to Kanas [5] and Kanas and Wiśniowska [8].

We also note that the function $p_{k}(z)=p(k, z)$ is continuous as regards $k, k \in[0, \infty)$ and has real coefficients for $k \in[0, \infty)$ (cf. $[4,5]$ ).

Let $\mathscr{P}$ denote the well-known Carathéodory class of functions, analytic in the unit disk with positive real part there, so that $\mathscr{P}=\{p: p$ analytic in $\mathcal{U}$, $p(0)=1, \operatorname{Re} p(z)>0\}$. Denote by $\mathscr{P}\left(p_{k}\right)$ the subclass of the class $\mathscr{P}$ consisting of all functions $p$ such that $p(U) \subset p_{k}(U)$.

Suppose that functions $g$ and $G$ are analytic in the unit disc $U$. The function $g$ is said to be subordinate to $G$, written $g \prec G$ (or $g(z) \prec G(z), z \in \mathcal{U}$ ), if $G$ is univalent in $U, g(0)=G(0)$, and $g(U) \subset G(U)$. If the function $g(z)=$ $\psi\left(p(z), z p^{\prime}(z)\right)$ with $\psi, p$ being analytic and having appropriate normalization, then the subordination is called the first-order differential subordination. The theory of differential subordinations was developed by Miller and Mocanu in their numerous works (cf., e.g., [12, 13, 14]) and by Eenigenburg et al. [1] and has wide applications in the theory of univalent functions.

We briefly recall one of the problems that characterizes this theory. For more details the reader should consult $[13,14]$. Assume that $p$ is analytic in $u$ and such that $p(0)=a$ and $p(z) \neq \equiv a$. Also, let $\psi: \mathbb{C}^{2} \mapsto \mathbb{C}$ be analytic and such that $\psi(p(0), 0)=a$. Given $\psi$ and univalent function $q, q(0)=p(0)$, find the "largest" domain $D$, such that $a \in D$, and the relation

$$
\psi\left(p(z), z p^{\prime}(z)\right) \in D \Longrightarrow p(z) \prec q(z)
$$

holds. The second important problem of this theory is finding the "smallest" function $q$, when $D$ and $\psi$ are given, and (1.4) holds.

Now, we recall some results from the theory of differential subordinations (cf., e.g., $[12,13,14]$ ) useful for further investigation (by $\boldsymbol{u}_{r}$ we denote the open disk centred at the origin and with the radius $r$ ).

LEMMA 1.1 (see [13]). Let $f$ be analytic in $u$, and let $g$ be analytic and univalent on $\bar{u}$, with $f(0)=g(0)$. If $f$ is not subordinate to $g$, then there exist points $z_{0} \in \mathcal{U}, \zeta_{0} \in \partial \mathcal{u}$, and $m \geq 1$ for which

$$
f\left(u_{\left|z_{0}\right|}\right) \subset g\left(u_{\left|z_{0}\right|}\right), \quad f\left(z_{0}\right)=g\left(\zeta_{0}\right), \quad z_{0} f^{\prime}\left(z_{0}\right)=m \zeta_{0} g^{\prime}\left(\zeta_{0}\right) .
$$

LEMMA 1.2 (see [13]). Let $\psi: \mathbb{C}^{2} \rightarrow \mathbb{C}$ be an analytic function in a domain $D \subset \mathbb{C}^{2}$, and let $p(z)=a+p_{n} z^{n}+\cdots$ be analytic in $u$, such that

$$
\psi\left(p(z), z p^{\prime}(z)\right) \prec h(z), \quad z \in U,
$$


where $h$ is analytic and univalent in $U$ with $\psi(p(0), 0)=h(0)$. If

$$
\psi\left(h\left(\zeta_{0}\right), m \zeta_{0} h^{\prime}\left(\zeta_{0}\right)\right) \notin h(\mathcal{U}) \quad \text { when } m \geq n, z \in U,\left|\zeta_{0}\right|=1 \text {, }
$$

then $p \prec h$ in $U$.

The univalent function $q$ is called a dominant of the solution of the differential subordination

$$
\psi\left(p(z), z p^{\prime}(z)\right) \prec h(z)
$$

or more simply a dominant if $p \prec q$ for all $p$ satisfying (1.8). A dominant $\tilde{q}$ that satisfies $\tilde{q} \prec q$ for all dominants $q$ of (1.8) is said to be the best dominant of (1.8). (Note that the best dominant is unique up to the rotation of $u$.)

LEMMA 1.3 (see $[1,12,14])$. Let $\beta$ and $\gamma$ be complex constants, and let $h$ be convex (univalent) in $U$, with $\operatorname{Re}(\beta h(z)+\gamma)>0$. Also, suppose that the differential equation

$$
q(z)+\frac{z q^{\prime}(z)}{\beta q(z)+\gamma}=h(z)
$$

has a univalent solution $q$, which satisfies $q \prec h$. If $p$ is analytic in $u$ with $p(0)=h(0)$ and satisfies

$$
p(z)+\frac{z p^{\prime}(z)}{\beta p(z)+\gamma} \prec h(z)
$$

then $p \prec q \prec h$ in $U$, and $q$ is the best dominant of (1.10).

LEMMA 1.4 (see [14, page 97]). Let $\beta, \gamma \in C, \beta \neq 0$, and $\operatorname{Re}(\beta+\gamma)>0$. Let $q$ be analytic in $U$, with $q(0)=h(0)=1$ and (1.9) is satisfied. If $\operatorname{Re}(\beta h(z)+\gamma)>0$ and $h$ is convex in $U$, then the solution $q$ of (1.10) is univalent and $q$ is the best dominant of (1.10).

In fact, the above lemmas appear in a more general form, but the above form is sufficient for our considerations. Lemmas 1.3 and 1.4 characterize properties of subordination, known as the subordination of the Briot-Bouquet type, described in detail by Miller and Mocanu in $[12,14]$ and by Eenigenburg et al. in [1].

Numerous subordination results concerning various choices of function $q$ and the domain $D$ in (1.4) appeared in the literature. Most of functions $q$ map the unit disk onto the right half-plane or more general half-plane, the disk and the sector contained in the right half-plane with the vertex at the origin (cf., e.g., [2, 3, 12, 13, 14]). The special case of the subordination related to conical domains did not receive the attention it deserves, but some results in this direction are known (cf. [6, 10]). In both papers only cases $\psi\left(p, z p^{\prime}\right)=$ $p+\gamma z p^{\prime} \prec h$, where function $h$ maps the unit disk onto the region bounded 
by parabolas and hyperbolas, were considered although the other forms of the function $\psi$ seem to be more useful. For instance, when we put $p(z)=$ $z f^{\prime}(z) / f(z)$ and $\psi\left(p, z p^{\prime}\right)=p+z p^{\prime} / p$, then the relation $\psi\left(p(z), z p^{\prime}(z)\right) \in$ $D \Rightarrow p(z) \prec q(z)$, for various choices of functions $q$ and $D$, gives inclusion relations between subclasses of convex and subclasses of starlike functions. Hence, in this case, the function $\psi$ in the form $\psi\left(p, z p^{\prime}\right)=p+\gamma z p^{\prime}$ is useless.

Our principal goal in this paper is to develop the theory of the first-order differential subordinations towards subordinations in conic domains. Such kind of domains is closely related to $k$-uniformly convex and $k$-starlike functions (cf. [7, 8, 9]). We employ geometric techniques to obtain certain differential inclusions when the functions $\psi$ and $q$ (or $\psi$ and a domain $D$ ) are given. Mainly, we discuss the case $\psi\left(p, z p^{\prime}\right)=p+z p^{\prime} / p$ and the function $h$ mapping the unit disk onto a domain bounded by a parabola.

In Section 2, we solve the problem of finding the largest domain $D$ for that, under given $\psi$ and $q$, the condition (1.4) is satisfied. The shape of the domain $D$ is described by the shape $q(\mathcal{U})$. In Section 3, we present the best dominant of the differential subordination (1.10). Various applications in the theory of univalent functions are given in Section 4.

2. Subordination results related to conic domains. Consider first the region bounded by a parabola $u=v^{2} / 2+1 / 2$ that is the domain $p_{1}$ ( $U$ ) with $p_{1}$ given by (1.2). Next, we consider the family described by the equality $u=$ $v^{2} / 2+(2 a+1) / 2, a<1 / 2$, that consists of parabolas with the vertex at $w=$ $(2 a+1) / 2$, symmetric about the real axis (our previous case corresponds to $a=0$ ). The family of domains containing point 1 inside and bounded by those parabolas may be characterized as

$$
\mathscr{D}_{a}=\{w: \operatorname{Re}(w-a)>|w-1-a|\},
$$

or equivalently

$$
\mathscr{D}_{a}=\left\{w=u+i v: 2 u>v^{2}+2 a+1\right\} \text {. }
$$

Note that $\mathscr{D}_{0}=\Omega_{1}$.

THEOREM 2.1. Let $p$ be a function analytic in the unit disk such that $p(0)=1$. Also, let $a<1 / 2$. If

$$
p(z)+\frac{z p^{\prime}(z)}{p(z)} \in \mathscr{D}_{a}
$$

then

$$
p(z) \prec 1+\frac{2}{\pi^{2}} \log ^{2} \frac{1+\sqrt{z}}{1-\sqrt{z}}:=p_{1}(z),
$$

where $\mathscr{D}_{a}$ is given by (2.1) and $a \geq a_{0}=-1 / \pi$. 
Proof. Suppose, on the contrary, that $p \nprec p_{1}$. Then, by Lemma 1.1, there exist points $z_{0} \in \mathcal{U}, \zeta_{0} \in \partial \mathcal{U}, \zeta_{0} \neq 1$, and $m \geq 1$ such that $p\left(z_{0}\right)=p_{1}\left(\zeta_{0}\right)$, $p\left(|z|<\left|z_{0}\right|\right) \subset p_{1}(u)$, and $z_{0} p^{\prime}\left(z_{0}\right)=m \zeta_{0} p_{1}^{\prime}\left(\zeta_{0}\right)$. Setting $p_{1}(z)=1+$ $\left(2 / \pi^{2}\right) \log ^{2} q(z)$ with $q(z)=(1+\sqrt{z}) /(1-\sqrt{z}), q(0)=1$, we obtain

$$
\frac{z p_{1}^{\prime}(z)}{p_{1}(z)}=\frac{4}{\pi^{2}} \frac{z q^{\prime}(z)}{q(z)} \frac{\log q(z)}{1+\left(2 / \pi^{2}\right) \log ^{2} q(z)}
$$

Note that for $\zeta_{0}=e^{i \theta}, \theta \in(0,2 \pi)$, we have $q\left(\zeta_{0}\right)=i \cot (\theta / 4)=i x, x>0$, and $\zeta_{0} q^{\prime}\left(\zeta_{0}\right) / q\left(\zeta_{0}\right)=i y=i(x+1 / x) / 4$. Thus

$$
\begin{aligned}
p\left(z_{0}\right) & =p_{1}\left(\zeta_{0}\right)=1+\frac{2}{\pi^{2}} \log ^{2}(i x)=\frac{1}{2}+\frac{2}{\pi^{2}} \log ^{2} x+i \frac{2}{\pi} \log x, \\
\frac{z_{0} p^{\prime}\left(z_{0}\right)}{p\left(z_{0}\right)} & =\frac{m \zeta_{0} p_{1}^{\prime}\left(\zeta_{0}\right)}{p_{1}\left(\zeta_{0}\right)} \\
& =\frac{4 m i y}{\pi^{2}} \frac{\log x+i(\pi / 2)}{(1 / 2)+\left(2 / \pi^{2}\right) \log ^{2} x+i(2 / \pi) \log x} \\
& =\frac{4 m y}{\pi^{2}}\left[\frac{(1 / \pi) \log x-(\pi / 4)+i \log x\left((3 / 2)+\left(2 / \pi^{2}\right) \log ^{2} x\right)}{\left((1 / 2)+\left(2 / \pi^{2}\right) \log ^{2} x\right)^{2}+\left(4 / \pi^{2}\right) \log ^{2} x}\right] .
\end{aligned}
$$

From the above we have

$$
\begin{aligned}
& \operatorname{Re}\left(p\left(z_{0}\right)+\frac{z_{0} p^{\prime}\left(z_{0}\right)}{p\left(z_{0}\right)}\right) \\
& \quad=\frac{1}{2}+\frac{2}{\pi^{2}} \log ^{2} x-m y \frac{\pi / 4-(1 / \pi) \log ^{2} x}{\log ^{2} x+\left(\pi / 4+(1 / \pi) \log ^{2} x\right)^{2}} \\
& \operatorname{Im}\left(p\left(z_{0}\right)+\frac{z_{0} p^{\prime}\left(z_{0}\right)}{p\left(z_{0}\right)}\right) \\
& \quad=\log x\left[\frac{2}{\pi}+m y \frac{3 / 2+\left(2 / \pi^{2}\right) \log ^{2} x}{\log ^{2} x+\left(\pi / 4+(1 / \pi) \log ^{2} x\right)^{2}}\right]
\end{aligned}
$$

Observe, by the definition of $\mathscr{D}_{a}$, that we will be led to a contradiction if we show that

$$
\operatorname{Im}^{2}\left(p\left(z_{0}\right)+\frac{z_{0} p^{\prime}\left(z_{0}\right)}{p\left(z_{0}\right)}\right)-2 \operatorname{Re}\left(p\left(z_{0}\right)+\frac{z_{0} p^{\prime}\left(z_{0}\right)}{p\left(z_{0}\right)}\right)+2 a+1 \geq 0,
$$


which, after necessary reductions, is equivalent to

$$
\begin{aligned}
m^{2} y^{2} \log ^{2} x \frac{\left(3 / 2+\left(2 / \pi^{2}\right) \log ^{2} x\right)^{2}}{\left(\log ^{2} x+\left(\pi / 4+(1 / \pi) \log ^{2} x\right)^{2}\right)^{2}} \\
+\frac{4 m}{\pi} y \log ^{2} x \frac{3 / 2+\left(2 / \pi^{2}\right) \log ^{2} x}{\log ^{2} x+\left(\pi / 4+(1 / \pi) \log ^{2} x\right)^{2}} \\
+2 m y \frac{\pi / 4-(1 / \pi) \log ^{2} x}{\log ^{2} x+\left(\pi / 4+(1 / \pi) \log ^{2} x\right)^{2}}+2 a \geq 0 .
\end{aligned}
$$

Set $t=\log ^{2} x, t \geq 0$. Then the above inequality can be rewritten as

$$
\begin{aligned}
m^{2} y^{2} t & \frac{\left(3 / 2+\left(2 / \pi^{2}\right) t\right)^{2}}{\left(t+(\pi / 4+(1 / \pi) t)^{2}\right)^{2}} \\
& +m y \frac{\left(8 / \pi^{3}\right) t^{2}+(4 / \pi) t+\pi / 2}{t+(\pi / 4+(1 / \pi) t)^{2}}+2 a \geq 0 .
\end{aligned}
$$

Since $m \geq 1$, "coefficients" of $y^{2}$ and $y$ are both nonnegative, and $y=(x+$ $1 / x) / 4=\left(e^{\sqrt{t}}+e^{-\sqrt{t}}\right) / 4=[\cosh \sqrt{t}] / 2$, condition (2.10) will be fulfilled if

$$
\begin{aligned}
\cosh ^{2} \sqrt{t} \frac{t\left(3 / 2+\left(2 / \pi^{2}\right) t\right)^{2}}{\left(t+(\pi / 4+(1 / \pi) t)^{2}\right)^{2}} \\
+2 \cosh \sqrt{t} \frac{\left(8 / \pi^{3}\right) t^{2}+(4 / \pi) t+(\pi / 2)}{t+(\pi / 4+(1 / \pi) t)^{2}}+8 a \geq 0 .
\end{aligned}
$$

Observe now that

$$
\begin{aligned}
\cosh ^{2} \sqrt{t} & \frac{t\left(3 / 2+\left(2 / \pi^{2}\right) t\right)^{2}}{\left(t+(\pi / 4+(1 / \pi) t)^{2}\right)^{2}} \\
+ & 2 \cosh \sqrt{t} \frac{\left(8 / \pi^{3}\right) t^{2}+(4 / \pi) t+\pi / 2}{t+(\pi / 4+(1 / \pi) t)^{2}}+8 a \\
\geq & 2 \frac{\left(8 / \pi^{3}\right) t^{2}+(4 / \pi) t+\pi / 2}{t+(\pi / 4+(1 / \pi) t)^{2}}+8 a \\
& =\frac{16}{\pi}\left[1-\frac{t}{t+(\pi / 4+(1 / \pi) t)^{2}}\right]+8 a \\
& \geq \frac{8}{\pi}+8 a .
\end{aligned}
$$

Indeed, the maximum of the function of $w(t)=t /\left[t+(\pi / 4+(1 / \pi) t)^{2}\right]$ on the interval $[0, \infty)$ is attained at $t_{0}=\pi^{2} / 4$ and equals $1 / 2$; from this the last 
inequality follows. Hence

$$
\operatorname{Im}^{2}\left\{p\left(z_{0}\right)+\frac{z_{0} p^{\prime}\left(z_{0}\right)}{p\left(z_{0}\right)}\right\}-2 \operatorname{Re}\left\{p\left(z_{0}\right)+\frac{z_{0} p^{\prime}\left(z_{0}\right)}{p\left(z_{0}\right)}\right\}+2 a+1 \geq 0
$$

if and only if $8 / \pi+8 a \geq 0$, that is, when $a \geq a_{0}=-1 / \pi \approx-0.318309$ that leads to the contradiction. The proof is completed.

Now, we extend the result of Theorem 2.1 to the case when $p(z)+$ $z p^{\prime}(z) /(\beta p(z)+\gamma) \in \mathscr{D}_{a}$ implies $p \prec p_{1}$. Since the method of the proof is the same as in Theorem 2.1, we omitted the details.

THEOREM 2.2. Let $\beta, \gamma \in \mathbb{R}$ be such that $\beta>\gamma \geq 0, \beta \neq 0, a<1 / 2$, and let $p \in \mathscr{P}$. If

$$
p(z)+\frac{z p^{\prime}(z)}{\beta p(z)+\gamma} \in \mathscr{D}_{a},
$$

then

$$
p(z) \prec 1+\frac{2}{\pi^{2}} \log ^{2} \frac{1+\sqrt{z}}{1-\sqrt{z}},
$$

where $\mathscr{D}_{a}$ is given by (2.1) and

$$
a \geq-\frac{2}{\pi \beta}+\frac{2}{\pi^{2}} r\left(t_{0}\right)=: a_{1},
$$

where

$$
\begin{aligned}
r(t) & =\frac{(2(2 \beta+\gamma) / \pi) t+\pi \gamma(\beta+2 \gamma) / 2 \beta}{\left(4 \beta^{2} / \pi^{2}\right) t+\left((\beta+2 \gamma) / 2+\left(2 \beta / \pi^{2}\right) t\right)^{2}}, \\
t_{0} & =\frac{\pi^{2}\left[2 \beta \sqrt{\beta(\beta+2 \gamma)\left(\beta^{2}-\gamma^{2}\right)}-\gamma(\beta+\gamma)\right]}{4 \beta(2 \beta+\gamma)} .
\end{aligned}
$$

Reasoning in the other direction we consider the function

$$
Q_{b}(z)=1+\frac{2(1-b)}{\pi^{2}} \log ^{2} \frac{1+\sqrt{z}}{1-\sqrt{z}}, \quad b<1,
$$

that maps the unit disk analytically and univalently onto the parabolic region $\widetilde{\Omega}_{b}=\left\{u+i v: 2 u>v^{2} /(1-b)+1+b\right\}$ having a vertex at $w=(1+b) / 2$. When $b$ grows, the parabola becomes narrower until it degenerates for $b=1$. The function $Q_{b}$ was defined by Rønning in [15]. Define now the family of domains, having 1 inside and creating from $\widetilde{\Omega}_{b}$ by a translation through the vector $(a, 0)$ as follows:

$$
\mathscr{D}_{a, b}=\left\{w=u+i v: 2(u-a)>\frac{v^{2}}{1-b}+1+b\right\}
$$


Reasoning along the same lines as in Theorem 2.1, in this case, we obtain the following theorem.

THEOREM 2.3. Let $a<(1-b) / 2, b<1$, and let $p$ be a function analytic in the unit disk such that $p(0)=1$. If

$$
p(z)+\frac{z p^{\prime}(z)}{p(z)} \in \mathscr{D}_{a, b},
$$

then $p(z) \prec Q_{b}(z)$, where $\mathscr{D}_{a, b}$ is given by (2.19), $Q_{b}(z)$ is given by (2.18), and

$$
a \geq-\frac{2}{\pi}+\frac{2(1-b)}{\pi^{2}} r\left(t_{0}\right)=: a_{2}
$$

where

$$
\begin{aligned}
r(t) & =\frac{(2(2-b) / \pi) t+\pi b(1+b) / 2(1-b)}{\left(4(1-b)^{2} / \pi^{4}\right) t^{2}+\left(2(1-b)(3+b) / \pi^{2}\right) t+(1+b)^{2} / 4}, \\
t_{0} & =\frac{\pi^{2}\left[\sqrt{(1-2 b)\left(1-b^{2}\right)}-b(1+b)\right]}{2(2-b)(1-b)} .
\end{aligned}
$$

3. Problem of the best dominant. The second problem of the theory of differential subordinations, mentioned in the introduction, is the problem of finding the "smallest" function $q$ such that (1.9) and (1.10) imply $p \prec q \prec h$. This problem was posed by Miller and Mocanu in [13], repeated among others in [14] and completely solved with reference to Briot-Bouquet differential subordination, although, an explicit form of the best dominant was found only for the functions mapping the unit disk onto a half-plane or a disk (given in term of hypergeometric functions). In this section, employing Miller and Mocanu results, we provide the form of the best dominant of Briot-Bouquet differential subordination in the case when the function $h \equiv p_{k}$.

In this instance we have the following proposition.

Proposition 3.1. Let $0 \leq k<\infty$. Also, let $\beta, \gamma \in \mathbb{C}$ be such that $\beta \neq 0$ and $\operatorname{Re}(\beta k /(k+1)+\gamma)>0$. If $p$ is analytic in $u, p(0)=1, p$ satisfies

$$
p(z)+\frac{z p^{\prime}(z)}{\beta p(z)+\gamma} \prec p_{k}(z),
$$

and $q$ is an analytic solution of

$$
q(z)+\frac{z q^{\prime}(z)}{\beta q(z)+\gamma}=p_{k}(z)
$$

then $q$ is univalent, $p \prec q \prec p_{k}$, and $q$ is the best dominant of (3.1).

PROoF. Since for each $k \in[0, \infty)$ the function $p_{k}$ is convex in $u$, and $\operatorname{Re}\left(\beta p_{k}(z)+\gamma\right)>0$, the assumptions of Lemma 1.4 are satisfied, that guarantees the univalence of a solution of the differential equality (3.2). Moreover, 
the solution $q$ is the best dominant of the subordination (3.1). Such a solution is given by

$$
q(z)=\frac{\left[z^{\gamma+\beta}\left(\exp \int_{0}^{z}\left(\left(p_{k}(t)-1\right) / t\right) d t\right)^{\beta}\right]}{\left[\beta \int_{0}^{z} u^{\beta+\gamma-1}\left(\exp \int_{0}^{u}\left(\left(p_{k}(x)-1\right) / x\right) d x\right)^{\beta} d u\right]}-\frac{\gamma}{\beta},
$$

or equivalently

$$
q(z)=\left[\beta \int_{0}^{1}\left(t^{\beta+\gamma-1} \exp \int_{z}^{t z} \frac{p_{k}(u)-1}{u} d u\right)^{\beta} d t\right]^{-1}-\frac{\gamma}{\beta}
$$

(cf. $[12,14]$ ). Thus, by making use of Lemma 1.3 , we have $p \prec q \prec p_{k}$ with $q$ being the best dominant.

In particular, for $\beta=1$ and $\gamma=0$, one obtains

$$
q(z)=\left[z \exp \int_{0}^{z} \frac{p_{k}(t)-1}{t} d t\right]\left[\int_{0}^{z}\left(\exp \int_{0}^{u} \frac{p_{k}(x)-1}{x} d x\right) d u\right]^{-1},
$$

or equivalently

$$
q(z)=\left[\int_{0}^{1}\left(\exp \int_{z}^{t z} \frac{p_{k}(u)-1}{u} d u\right) d t\right]^{-1} .
$$

In the case when $k>1$ we may approximate the domain, being an elliptical domain, by a circular domain. An ellipse must be contained in the disk which is tangent to the ellipse at points common with the real axis. The case of subordinations related to disks is completely solved in the literature. The first attempt was given by Jakubowski and Kamiński [3], but a final result is due to Miller and Mocanu (cf., e.g., [14, page 110]). Since the ellipse intersects the real axis at points $k /(k+1)$ and $k /(k-1)$, then such a circle $K(S, R)$ has the center at $S=k^{2} /\left(k^{2}-1\right)$ and a radius $R=k /\left(k^{2}-1\right)$ and contains the point $z=1$ inside. Then the function $\varphi: \vartheta \rightarrow K(S, R)$ has a form $\varphi(z)=k /(k-z)$, and therefore Proposition 3.1 with $\beta=1$ and $\gamma=0$ yields the following proposition.

Proposition 3.2. Let $1<k<\infty$ and let $p$ be analytic in $u, p(0)=1$, and $p$ satisfies (3.1). Then $p(z) \prec z /[(z-k) \log (1-z / k)]$ and

$$
\operatorname{Re} p(z)>\frac{1}{(k+1) \log (1+1 / k)}
$$

Proof. Since $p$ satisfies (3.1) and for each fixed $k, p_{k}(z) \prec k /(k-z)$, then $p(z)+z p^{\prime}(z) / p(z) \prec k /(k-z)$ in $u$. In view of (3.6), inserting $\varphi(z)=k /(k-z)$ in place of $p_{k}$, we obtain $p(z) \prec q(z)=z /[(z-k) \log (1-z / k)]$. Then we have $q(-1)=1 /[(k+1) \log (1+1 / k)]$, and the assertion follows. 
REMARK 3.3. Making use of Proposition 3.2, for instance, for the case $k=2$, one obtains that if $p(z)+z p^{\prime}(z) / p(z) \prec p_{2}(z)=1 /(1-z / 2)$ (it is obvious that then $\left.\operatorname{Re}\left(p(z)+z p^{\prime}(z)^{\prime} p(z)\right)>2 / 3\right)$ implies $\operatorname{Re} p(z)>1 /[3 \log (3 / 2)] \approx$ $0.813 \ldots$.

4. Applications. Functions $p_{k}$ and the class $\mathscr{P}\left(p_{k}\right)$ were constructed with reference to the subclass of the class of univalent functions, called $k$-uniformly convex, and denoted $k$ - $\mathfrak{U}_{\mathfrak{C}} \mathrm{V}$ (cf. [7, 8, 9]), so that every domain appeared in Section 2 is strictly related with the class $\mathscr{P}\left(p_{k}\right)$ and, obviously, with $k$ - $U \mathscr{C} \mathscr{V}$. It should be mentioned that the class $k$ - $U \mathscr{C} \mathscr{V}$ was defined pure geometrically as the class of such functions that map every circular arc, with the center $\zeta,|\zeta| \leq k,(0 \leq k<\infty)$ contained in $U$, onto a convex arc. With reference to the class $k$ - $u \mathscr{C} \mathscr{V}$, Kanas and Wiśniowska defined the class $k-\mathscr{Y} \mathscr{T}=\{g \in \mathscr{H}: \mathfrak{g}=$ $z f^{\prime}, f \in k$ - $\left.u \mathscr{C} \mathscr{V}\right\}$, called the class of $k$-starlike functions. Here $\mathscr{H}$ denotes the class of functions analytic in the unit disk and such that $f(0)=f^{\prime}(0)-1=0$.

One of the most important cases of $k-\mathcal{U} \mathscr{C}^{\mathscr{V}} \mathfrak{V}$ functions is the uniformly convex functions case which corresponds to the choice $k=1$. In this case, condition (1.1), when setting $u+i v=1+z f^{\prime \prime}(z) / f^{\prime}(z), z \in U$, means that the range of the expression $1+z f^{\prime \prime}(z) / f^{\prime}(z)$ is the region bounded by a parabola $u=$ $v^{2} / 2+1 / 2$.

THEOREM 4.1. Let $f \in \mathscr{H}_{\text {and }}$ let $1+z f^{\prime \prime}(z) / f^{\prime}(z) \in \mathscr{D}_{a}$, where $\mathscr{D}_{a}$ given in (2.1) and $a \geq-1 / \pi$. Then

$$
\frac{z f^{\prime}(z)}{f(z)} \prec 1+\frac{2}{\pi^{2}} \log ^{2} \frac{1+\sqrt{z}}{1-\sqrt{z}}
$$

or equivalently $f \in 1$ - $9 \mathcal{G}$.

Proof. Assume that $1+z f^{\prime \prime}(z) / f^{\prime}(z) \in \mathscr{D}_{a}$. Setting $p(z)=z f^{\prime}(z) / f(z)$, we have $p(0)=1$, and the above condition can be rewritten as

$$
p(z)+\frac{z p^{\prime}(z)}{p(z)} \in \mathscr{D}_{a}
$$

in $\mathcal{U}$. Now, applying Theorem 2.1, we conclude the assertion.

Applying Theorem 2.2 and proving similarly as above, we obtain the following theorem.

THEOREM 4.2. Let $\beta, \gamma \in \mathbb{R}, \beta \neq 0, \beta>\gamma \geq 0$, and let $f \in \mathscr{H}$. If

$$
\frac{1+z f^{\prime \prime}(z) / f^{\prime}(z)-z f^{\prime}(z) / f(z)}{\beta+\gamma\left(f(z) / z f^{\prime}(z)\right)} \in \mathscr{D}_{a},
$$

where $\mathscr{D}_{a}$ is given in (2.1) and a satisfies (2.16), then $f \in 1-\mathscr{Y T}$.

Making use of Theorem 2.3 by a simple substitution, like in the proof of Theorem 4.1, we have the following theorem. 
THEOREM 4.3. Let $a<1 / 2$, and $b<1$. Also, let $f \in \mathscr{H}$ and $1+z f^{\prime \prime}(z) / f^{\prime}(z) \in$ $\mathscr{D}_{a, b}$, where $\mathscr{D}_{a, b}$ is given by (2.19) and $a \geq a_{1}$ (the value $a_{1}$ is described by (2.21)). Then $z f^{\prime}(z) / f(z) \prec Q_{b}(z)$ with $Q_{b}$ given by (2.18).

Conversely to the presented applications we may assume that

$$
f \in k-\mathcal{U} \mathscr{G} \mathfrak{V} \Longleftrightarrow 1+\frac{z f^{\prime \prime}(z)}{f^{\prime}(z)} \prec p_{k}(z),
$$

and from this, by making use of results of Section 3, we conclude the containment results about $k$ - $\mathscr{T} \mathcal{F}$.

On applying Proposition 3.1 (for $\beta=1$ and $\gamma=0$ ) and Proposition 3.2, we have the following relations between the classes $k-\mathcal{U} \mathscr{G} \mathscr{V}$ and $k$ - $\mathscr{T} \mathcal{T}$ below.

Proposition 4.4. Let $0 \leq k<\infty$. Also, let $f \in k-\mathcal{U} \mathscr{C}^{\mathfrak{V}}$. Then $z f^{\prime}(z) / f(z) \prec$ $q(z) \prec p_{k}(z)$, where $q$ is given by (3.6).

Proposition 4.5. Let $1<k<\infty$ and let $f \in k$-UG $\mathscr{V}$. Then $z f^{\prime}(z) / f(z) \prec$ $z /[(z-k) \log (1-z / k)]$ and

$$
\operatorname{Re} \frac{z f^{\prime}(z)}{f(z)}>\frac{1}{(k+1) \log (1+1 / k)} .
$$

We presented applications of results of Sections 3 and 4 only for $k-\mathcal{U} \mathscr{C} V$ and $k$ - $\mathscr{Y} \mathcal{T}$ functions. However, by setting $p(z)=f(z) / z, p(z)=f^{\prime}(z), p(z)=$ $2 f(z) / z-1, p(z)=2 z f^{\prime}(z) / f(z)-1, p(z)=2 \sqrt{f^{\prime}(z)}-1$, and so forth, in the propositions and the theorems of the mentioned sections, we may obtain many more results related to conical domains.

\section{REFERENCES}

[1] P. Eenigenburg, S. S. Miller, P. T. Mocanu, and M. O. Reade, On a Briot-Bouquet differential subordination, General Inequalities, 3 (Oberwolfach, 1981), Internat. Schriftenreihe Numer. Math., vol. 64, Birkhäuser, Basel, 1983, pp. 339348.

[2] D. J. Hallenbeck and S. Ruscheweyh, Subordination by convex functions, Proc. Amer. Math. Soc. 52 (1975), 191-195.

[3] Z. J. Jakubowski and J. Kamiński, On some properties of Mocanu-Janowski functions, Rev. Roumaine Math. Pures Appl. 23 (1978), no. 10, 1523-1532.

[4] S. Kanas, Coefficients estimates in subclasses of the Carathéodory class related to conical domains, to appear in Acta Sci. Math. (Szeged).

[5] _ Subclasses of the Carathéodory class related to conical domains, to appear in Georgian Math. J.

[6] S. Kanas and A. Lecko, Differential subordination for domains bounded by hyperbolas, Zeszyty Nauk. Politech. Rzeszowskiej Mat. 175 (1999), no. 23, 61-70.

[7] S. Kanas and A. Wiśniowska, Conic regions and k-uniform convexity. II, Zeszyty Nauk. Politech. Rzeszowskiej Mat. 170 (1998), no. 22, 65-78.

[8] Conic regions and k-uniform convexity, J. Comput. Appl. Math. 105 (1999), no. 1-2, 327-336. 
[9] _ Conic domains and starlike functions, Rev. Roumaine Math. Pures Appl. 45 (2000), no. 4, 647-657.

[10] Y. Ch. Kim and A. Lecko, On a differential subordination for domains bounded by parabolas, Proc. Japan Acad. Ser. A Math. Sci. 75 (1999), no. 9, 163-165.

[11] W. C. Ma and D. Minda, Uniformly convex functions, Ann. Polon. Math. 57 (1992), no. $2,165-175$.

[12] S. S. Miller and P. T. Mocanu, Univalent solutions of Briot-Bouquet differential equations, J. Differential Equations 56 (1985), no. 3, 297-309.

[13] _ The theory and applications of second-order differential subordinations, Studia Univ. Babeş-Bolyai Math. 34 (1989), no. 4, 3-33.

[14]_, Differential Subordinations. Theory and Applications, Monographs and Textbooks in Pure and Applied Mathematics, vol. 225, Marcel Dekker, New York, 2000.

[15] F. Rønning, On starlike functions associated with parabolic regions, Ann. Univ. Mariae Curie-Skłodowska Sect. A 45 (1991), 117-122.

[16] - Uniformly convex functions and a corresponding class of starlike functions, Proc. Amer. Math. Soc. 118 (1993), no. 1, 189-196.

Stanisława Kanas: Department of Mathematics, Rzeszów University of Technology, W. Pola 2, Pl-35-959 Rzeszów, Poland

E-mail address: skanas@prz.edu.p1 


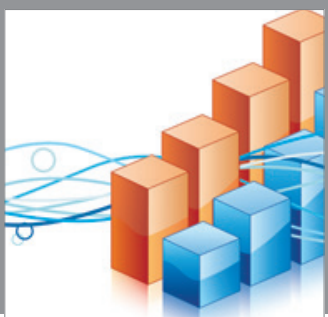

Advances in

Operations Research

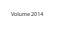

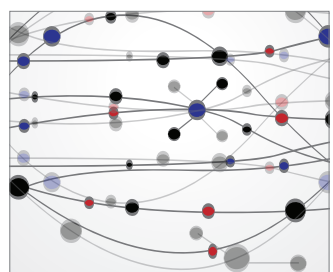

\section{The Scientific} World Journal
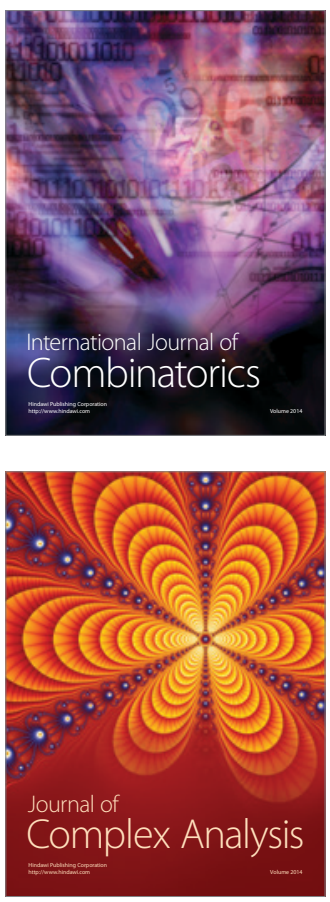

International Journal of

Mathematics and

Mathematical

Sciences
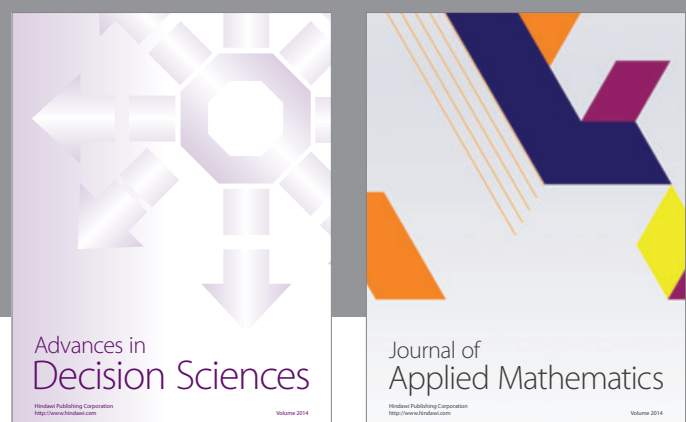

Journal of

Applied Mathematics
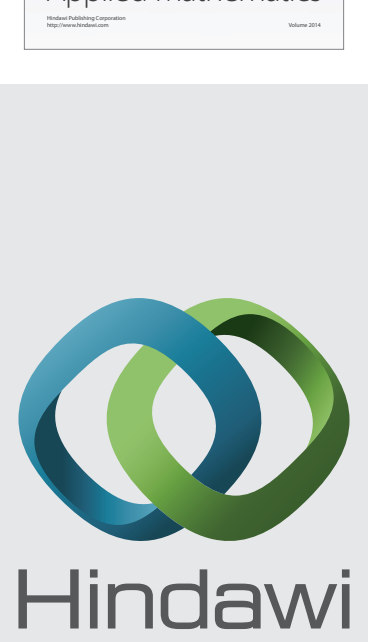

Submit your manuscripts at http://www.hindawi.com
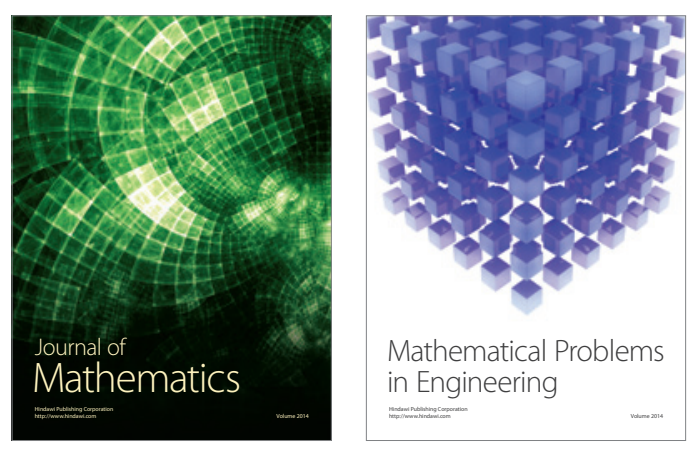

Mathematical Problems in Engineering
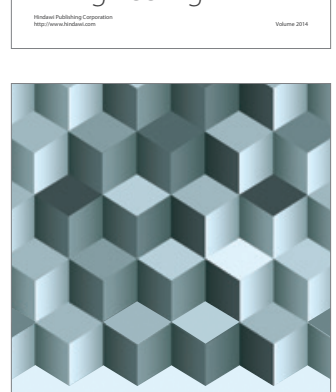

Journal of

Function Spaces
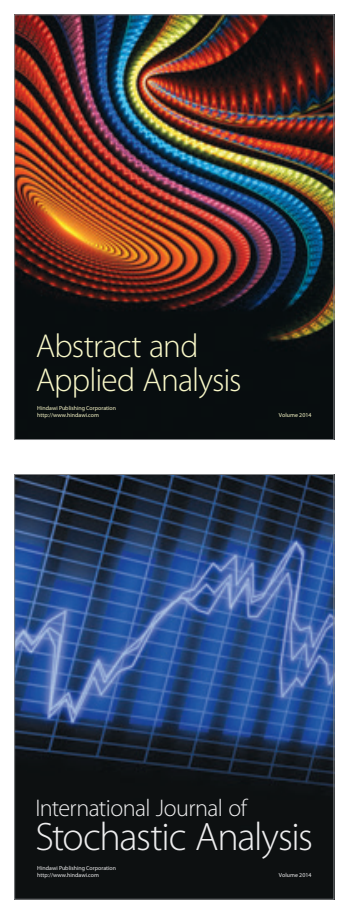

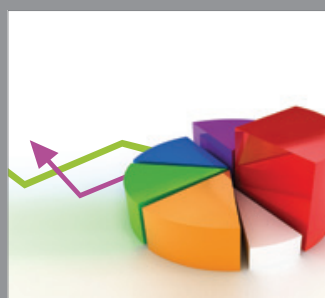

ournal of

Probability and Statistics

Promensencen
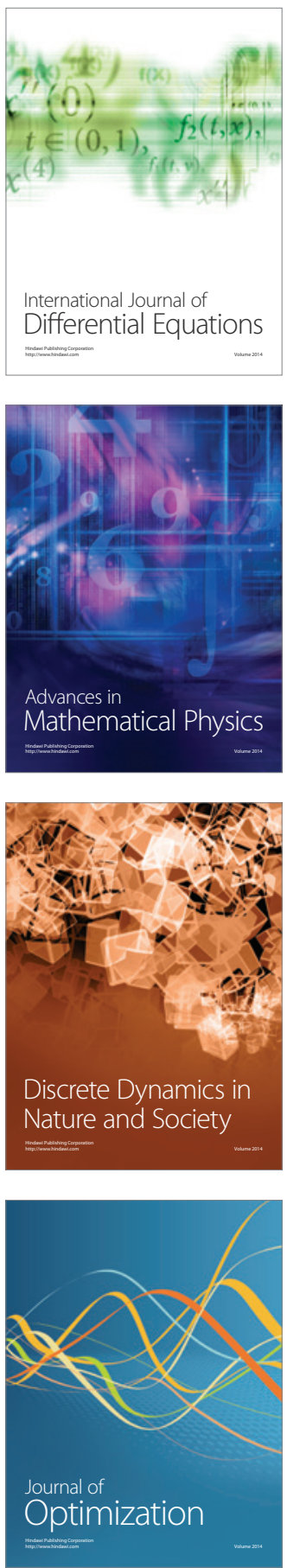